\title{
Application of the UHREM Technique in Atomic Modelling of Growth Defects in CVD-Diamond Films
}

\author{
D. Dorignac
}

\author{
CNRS, Centre d'Elaboration de Matériaux et d'Etudes Structurales, 31055 Toulouse, France
}

Diamond is of course known not only as the stuff of women's dreams - since at least two thousand years before Christ, "Diamonds have been a girl's best friend" - but also for its unique physicochemical properties. When considering any characteristic property of a material, the value associated with diamond almost always corresponds to an extremum among all materials considered for that property. Diamond is effectively the hardest known material, with the highest Young's modulus, the highest sound velocity, a high wear resistance and a very low coefficient of friction. It is the best thermal conductor of any material, with a superior thermal shock resistance and a very low coefficient of expansion. It is transparent over a very broad spectral range, from X-rays to the far IR, with a low refractive index in the visible. It is chemically inert and highly resistant to acids. Diamond is also a very good electrical insulator, with wide bandgap, very high electrical breakdown strength, near zero workfunction, negative electron affinity, UV photoconductivity and it has the lowest dielectric constant of all the ceramic materials. And, finally, it can be doped to form $\mathrm{p}$ - and ntype semiconductors, which present the highest hole mobility and the highest saturated electron velocity, leading to the highest figures of merit in electronics, which exceed by far those of other semiconductors.

The above long-recognized combination of superlative properties with the recent chemical-vapourdeposition (CVD) ability to grow diamond as a film, which began in the 1980s and only really emerged in the 1990s, makes diamond an exceptional material for a wide variety of industrial uses and a vast range of potential applications. Much of the relevant literature can be found in references [1-5]. Typical applications can be classified, based on the range of extremely desirable properties of diamond, as follows: high hardness $\Rightarrow$ tooling; low chemical reactivity $\Rightarrow$ high-performance structural materials. But, noting that diamond can very efficiently transport, transmit or convert energy, and that its interactions in various ways with the entire electromagnetic spectrum can be valuable, it is much more instructive to look at these applications from the perspective of the energy spectrum. The main application areas and product classes are then found to be, in order of increasing energy: (i) phonons $\Rightarrow$ thermal management structures, sensors, surface-acoustic-wave devices; (ii) microwaves $\Rightarrow$ radomes, waveguides; (iii) photons $\Rightarrow$ IR and laser windows, optical switches and emitters, displays, UV detectors and focal plane arrays, X-ray windows and masks; and finally (iv) electrons $\Rightarrow$ high speed-, high temperature-, high power-, radiation hard-electronics (diodes, transistors and integrated circuits), and also capacitors and vacuum microelectronics (cold cathode components). It can be concluded that when ultimate properties are required, CVD-diamond is the ultimate answer, particularly in the semiconductor field because no other substance performs under duress as well as diamond. It is thus thought that diamond will become a widely used material in this 21 st century, resourcefully engineered into high-value-added components and systems.

This highlights the importance of technology for controlling the purity, crystallinity and defect structure in diamond as required for each particular performance criterion. During the past decade, the growth defects present in this promising engineering material have thus been studied by high 
resolution electron microscopy with a view to understanding their crucial role in influencing its practical properties. Several investigations of the defect structure have been performed using microscopes with point-resolutions down to 0.17-0.16 $\mathrm{nm}$ [6-18] and down to 0.12-0.10 nm [19-24], since detailed atomic resolution images can effectively provide local crystallographic information on the defect core structures. The most striking feature observed in such films is the presence of multiple twinning on $\{111\}$ planes. The twins are frequently present as large domains, the simplest interfaces corresponding to coherent first order $(\Sigma=3)$ twinning. Higher order interfaces $\left(\Sigma=3^{\mathrm{n}}, \mathrm{n}=2\right.$ to 4), pentagonal arrangements of twins, twinning dislocations, stacking faults and different types of associated dislocations have also been identified. My emphasis will thus be on the use of ultra-highresolution electron microscopy (UHREM) at $0.12 \mathrm{~nm}$ to characterize, at the atomic level, the defects present in CVD-diamond. Plausible 3D atomic-scale models can be proposed, based on imagematching with computer simulations and built with reasonable compression-dilation of the bond lengths and distortions of the tetrahedral angles, according to recent self-consistent $a b$ initio calculations of minimum-energy defect structures. From these models, evidence for unusual structural units, which are believed to be quite characteristic of the CVD-diamond growth, has sometimes emerged. Since CVD-diamond is expected to find gradually increasing acceptance in industrial applications during the 21 st century, I hope that the UHREM technique will help researchers in this field in their endeavours to create deposition areas with a high degree of uniformity in terms of growth rate and material quality.

\section{References}

[1] K. E. Spear and J. P. Dismukes, Synthetic Diamond, Wiley, New York, 1994.

[2] D. M. Gruen and I. Buckley-Golder, Special Issue of MRS Bulletin 23 (9) (1998).

[3] B. Dischler and C. Wild, Low-pressure Synthetic Diamond, Springer, Berlin, 1998.

[4] M.A. Prelas et al., Handbook of Industrial Diamonds, Marcel Dekker, New York, 1998.

[5] P.W. May, Phil. Trans. R. Soc. Lond. A 358 (2000) 473.

[6] W. Luyten et al., Phil. Mag. 66 (1992) 899.

[7] D. Shechtman et al., J. Mater. Res. 8 (1993) 473.

[8] M. Joksch et al., Diamond Relat. Mater. 3 (1994) 681.

[9] D. Shechtman, Mat. Sci. Engn. A 184 (1994) 113.

[10] C. J. Chen et al., J. Mater. Res. 10 (1995) 3041.

[11] X. Jiang and C. L. Jia, Appl. Phys. Lett. 67 (1995) 1197.

[12] C. L. Jia et al., Phys. Rev. B 52 (1995) 5164.

[13] C. J. Chen et al., J. Mater. Res. 11 (1996) 1002.

[14] H. Verhoeven et al., Appl. Phys. Lett. 71 (1997) 1329.

[15] D. Wittorf et al., Diamond Relat. Mater. 6 (1997) 649; 9 (2000) 1696.

[16] H. Ichinose and M. Nakanose, Thin Solid Films 319 (1998) 87.

[17] Y. Zhang et al., J. Electron. Microsc. 48 (1999) 245.

[18] L.C. Nistor et al., Phys. Stat. Sol. 174 (1999) 5; 186 (2001) 207.

[19] D. Dorignac et al., Diamond Relat. Mater. 6 (1997) 758.

[20] S. Delclos et al., Diamond Relat. Mater. 7 (1998) 222; 8 (1999) 682; 9 (2000) 346.

[21] H. Sawada et al., Diamond Relat. Mater. 10 (2001) 2030.

[22] H. Sawada and H. Ichinose, Scripta Mater. 44 (2001) 2327.

[23] D. Dorignac et al., J. Phys. IV France 11 (2001) 971.

[24] D. Dorignac et al., Phil. Mag. B 81 (2001) 1879. 